\title{
SHIMURA SUBGROUPS OF JACOBIANS OF SHIMURA CURVES
}

\author{
SAN LING \\ (Communicated by William W. Adams)
}

\begin{abstract}
Given an indefinite quaternion algebra of reduced discriminant $D$ and an integer $N$ relatively prime to $D$, one can construct Shimura curves $\mathrm{Sh}_{0}(N, D)$ and $\operatorname{Sh}_{1}(N, D)$, which are analogues of $X_{0}(N)$ and $X_{1}(N)$. The natural morphism $\operatorname{Sh}_{1}(N, D) \rightarrow \mathrm{Sh}_{0}(N, D)$ induces a morphism $J_{0}(N, D) \rightarrow$ $J_{1}(N, D)$ between the Jacobians. We compute the kernel $\Sigma(N, D)$ of this latter map, which is finite.
\end{abstract}

Let $\mathscr{B}$ be an indefinite quaternion algebra over $\mathbf{Q}$ that is ramified at an even number of primes $l_{1}, \ldots, l_{n}$. Then $\mathscr{B}$ has reduced discriminant $D=\prod_{i} l_{i}$. Let $N \geq 1$ be an integer that is relatively prime to $D$. Suppose that $p$ is a prime dividing $N$. Let

The algebra $\mathscr{B}_{p} \stackrel{\text { def }}{=} \mathscr{B} \otimes \mathbf{Q}_{p}$ is isomorphic to the matrix algebra $M_{2}\left(\mathbf{Q}_{p}\right)$.

$$
\mathscr{O}_{p, m}=\left(\begin{array}{cc}
\mathbf{Z}_{p} & \mathbf{Z}_{p} \\
p^{m} \mathbf{Z}_{p} & \mathbf{Z}_{p}
\end{array}\right)
$$

be the canonical Eichler order of level $p^{m} \mathbf{Z}_{p}$ in $M_{2}\left(\mathbf{Q}_{p}\right)$. We define the groups $\Gamma_{0}\left(p^{m}, \mathbf{Z}_{p}\right)$ and $\Gamma_{1}\left(p^{m}, \mathbf{Z}_{p}\right)$ to be

$$
\begin{gathered}
\Gamma_{0}\left(p^{m}, \mathbf{Z}_{p}\right)=\mathrm{SL}_{2}\left(\mathbf{Z}_{p}\right) \cap \mathscr{O}_{p, m}, \\
\Gamma_{1}\left(p^{m}, \mathbf{Z}_{p}\right)=\left\{x \in \Gamma_{0}\left(p^{m}, \mathbf{Z}_{p}\right) \mid x \equiv\left(\begin{array}{cc}
1 & * \\
0 & 1
\end{array}\right) \bmod p^{m} \mathscr{O}_{p, 0}\right\} .
\end{gathered}
$$

Let $\mathscr{O}$ be a Z-Eichler order in $\mathscr{B}$ of level $N$, and let $p^{m}$ be the exact power of $p$ dividing $N$. For such a $p$, let $i_{p}: \mathbf{Q} \hookrightarrow \mathbf{Q}_{p}$ be the canonical embedding. This embedding can be extended to an embedding, also denoted by $i_{p}$, of $\mathscr{B}$ in $M_{2}\left(\mathbf{Q}_{p}\right)$, such that $i_{p}(\mathscr{O})$ is the canonical Eichler order $\mathscr{O}_{p, m}$ of level $p^{m} \mathbf{Z}_{p}$ [4, pp. 108-109]. The inverse image of $\Gamma_{0}\left(p^{m}, \mathbf{Z}_{p}\right)$ under $i_{p}$ is $\mathscr{O}^{1}$, the set of elements of $\mathscr{O}$ of (reduced) norm 1 . We define the congruence subgroups $\Gamma_{0}(N, D)$ and $\Gamma_{1}(N, D)$ as

$$
\begin{aligned}
& \Gamma_{0}(N, D)=\left\{x \in \mathscr{O}^{1} \mid i_{p}(x) \in \Gamma_{0}\left(p^{m}, \mathbf{Z}_{p}\right) \text { for all } p \mid N\right\}, \\
& \Gamma_{1}(N, D)=\left\{x \in \mathscr{O}^{1} \mid i_{p}(x) \in \Gamma_{1}\left(p^{m}, \mathbf{Z}_{p}\right) \text { for all } p \mid N\right\} .
\end{aligned}
$$

Received by the editors October 16, 1991.

1991 Mathematics Subject Classification. Primary 11G18.

This research is financially supported by the National University of Singapore Overseas Graduate Scholarship. 
Since $\mathscr{B}$ is unramified at the infinite place of $\mathbf{Q}$, it follows that $\mathscr{B} \otimes_{\mathbf{Q}} \mathbf{R}$ is isomorphic to $M_{2}(\mathbf{R})$. Upon fixing such an isomorphism, we obtain an embedding $i_{\infty}: \mathscr{O}^{1} \hookrightarrow \mathrm{SL}_{2}(\mathbf{R})$. The action of $\mathrm{SL}_{2}(\mathbf{R})$ on the Poincare upper half plane $\mathscr{H}=\{\tau \in \mathbf{C} \mid \operatorname{Im}(\tau)>0\}$

$$
\left(\left(\begin{array}{ll}
a & b \\
c & d
\end{array}\right), \tau\right) \mapsto \frac{a \tau+b}{c \tau+d}
$$

implies that the groups $\Gamma_{i}(N, D)(i=0,1)$ also act on $\mathscr{H}$. When $D>1$, the quotient $\{ \pm 1\} \Gamma_{i}(N, D) \backslash \mathscr{H}(i=0,1)$ has a natural structure of compact connected Riemann surface. It is called a Shimura curve, which we shall denote by $\operatorname{Sh}_{i}(N, D)$. (When $D=1$, the quaternion algebra $\mathscr{B}$ is the matrix algebra $M_{2}(\mathbf{Q})$. The quotients $\{ \pm 1\} \Gamma_{i}(N, 1) \backslash \mathscr{H}(i=0,1)$ are known to be noncompact. Their compactifications are the classical modular curves $X_{i}(N)$ $(i=0,1)$ described in the introduction of [2].)

From the inclusion $\Gamma_{1}(N, D) \subseteq \Gamma_{0}(N, D)$, we get a natural morphism of curves $u: \operatorname{Sh}_{1}(N, D) \rightarrow \operatorname{Sh}_{0}(N, D)$.

Let $J_{i}(N, D)$ denote the Jacobian of $\operatorname{Sh}_{i}(N, D)$. Let

$$
u^{*}: J_{0}(N, D) \rightarrow J_{1}(N, D)
$$

be the morphism of abelian varieties deduced from $u$ via Pic functoriality. Its kernel, called the Shimura subgroup, is a finite group; we denote it by $\Sigma(N, D)$.

The Shimura subgroup $\Sigma(N, D)$ when $D=1$ (i.e., in the case of classical modular curves) is studied in [3] when $N$ is prime and in [2] for arbitrary $N$. In this note, we study $\Sigma(N, D)$ for $D>1$.

As in the case of classical modular curves, we see readily that the morphism $u: \operatorname{Sh}_{1}(N, D) \rightarrow \operatorname{Sh}_{0}(N, D)$ is a Galois covering with Galois group $\Gamma_{0}(N, D) /\{ \pm\} \Gamma_{1}(N, D)$. We can identify $\Gamma_{0}(N, D) / \Gamma_{1}(N, D)$ with $(\mathbf{Z} / N \mathbf{Z})^{\times}$ as follows.

For $x \in \Gamma_{0}(N, D)$, let

$$
i_{p}(x)=\left(\begin{array}{ll}
a_{p}(x) & b_{p}(x) \\
c_{p}(x) & d_{p}(x)
\end{array}\right) \in \Gamma_{0}\left(p^{m}, \mathbf{Z}_{p}\right) .
$$

Consider the reduction $d_{p}(x) \in\left(\mathbf{Z}_{p} / p^{m} \mathbf{Z}_{p}\right)^{\times} \simeq\left(\mathbf{Z} / p^{m} \mathbf{Z}\right)^{\times}$. By the Chinese Remainder Theorem, there exists a unique $d(x) \in(\mathbf{Z} / N \mathbf{Z})^{\times}$such that $d(x) \equiv$ $d_{p}(x) \bmod p^{m}$ for all $p \mid n$. The homomorphism $d: \Gamma_{0}(N, D) \rightarrow(\mathbf{Z} / N \mathbf{Z})^{\times}=$ $\prod\left(\mathbf{Z} / p^{m} \mathbf{Z}\right)^{\times}$, given by $x \mapsto d(x)$, is surjective and clearly has kernel $\Gamma_{1}(N, D)$. Hence,

$$
\Gamma_{0}(N, D) / \Gamma_{1}(N, D) \simeq(\mathbf{Z} / N \mathbf{Z})^{\times} .
$$

Let $\mathbf{U}$ be the group of complex numbers of modulus 1 . We prove in $\S 1$ the main theorem of this paper.

Theorem 1. The Shimura subgroup $\Sigma(N, D)$ of $J_{0}(N, D)$ is canonically isomorphic to the group of homomorphisms $g:(\mathbf{Z} / N \mathbf{Z})^{\times} \rightarrow \mathbf{U}$ such that $g(d)=1$ 
if $d=-1$ or $d^{2}+t d+1=0$, where

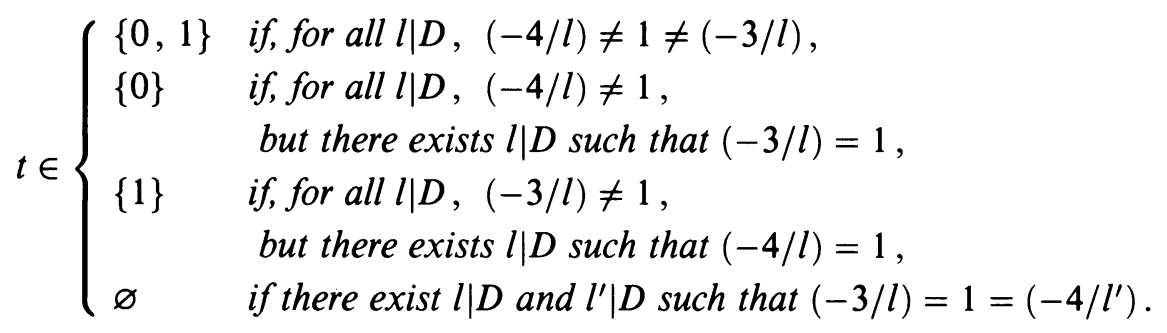

Corollary 1. Let $D>1$, let $\phi(N)$ denote the number of elements of $(\mathbf{Z} / N \mathbf{Z})^{\times}$, and:

(i) let $k$ be the number of prime divisors of $N$ distinct from 2 and 3;

(ii) let $m_{2}$ be equal to 2 if, for all $l$ dividing $D,(-4 / l) \neq 1$, and -1 is a square $\bmod N$ (i.e., if $4 \nmid N$ and each prime factor $p \neq 2$ of $N$ is congruent to $1 \mathrm{mod} 4)$, and let $m_{2}$ be equal to 1 otherwise;

(iii) let $m_{3}$ be equal to 3 if, for all $l$ dividing $D,(-3 / l) \neq 1$, and $X^{2}+X+1$ has a root $\bmod N$ (i.e., if $9 \nmid N$ and each prime factor $p \neq 3$ of $N$ is congruent to $1 \bmod 3)$, and let $m_{3}$ be equal to 1 otherwise.

Then we have

$$
\operatorname{Card}(\Sigma(N, D))= \begin{cases}\phi(N) /\left(2 m_{2}^{k} m_{3}^{k}\right) & \text { if } N \geq 5, \\ 1 & \text { if } N \leq 4 .\end{cases}
$$

Corollary 2. Let $D>1$, let $N=\prod p^{r_{p}}$ be the prime power decomposition of $N$, and:

(i) let $r_{p}^{\prime}$ be equal to $r_{p}-1$ if $p \neq 2$;

(ii) let $r_{2}^{\prime}$ be equal to $\max \left(0, r_{2}-2\right)$;

(iii) let $e_{0}$ be equal to $\operatorname{lcm}_{p \mid N}\left((p-1) p^{r_{p}^{\prime}}\right)$;

(iv) let $m_{1}$ be equal to 2 if $N$ is the product of 1 or 2 by a power of an odd prime, and let $m_{1}$ be equal to 1 otherwise;

(v) let $m_{2}$ and $m_{3}$ be as in Corollary 1.

Then the exponent of the group $\Sigma(N, D)$ (i.e., the smallest integer $e$ such that $e \Sigma(N, D)=0)$ is given by

$$
e= \begin{cases}e_{0} /\left(m_{1} m_{2} m_{3}\right) & \text { if } N \geq 5, \\ 1 & \text { if } N \leq 4 .\end{cases}
$$

Corollary 3. For $D>1$, the only integers $N$ for which the order of $\Sigma(N, D)$ is 1 are

$$
\begin{cases}1,2,3,4,6 & \text { for any } D>1, \\ 5,10 & \text { if, for all } l \mid D,(-4 / l) \neq 1 \\ 7 & \text { if, for all } l \mid D,(-3 / l) \neq 1, \\ 13 & \text { if, for all } l \mid D,(-3 / l) \neq 1 \neq(-4 / l) .\end{cases}
$$

Corollary 4. When $N$ approaches infinity, the exponent and a fortiori the order of $\Sigma(N, D)$ go to infinity.

\section{Proof of TheOREM 1}

Let $\Gamma$ be a discrete subgroup of $\mathrm{SL}_{2}(\mathbf{R})$ of finite covolume. Suppose that the Riemann surface $X=\Gamma \backslash \mathscr{H}$ is compact and let $J(X)$ be the Jacobian. Then 
the argument in $[2, \S 1.5]$ produces a canonical injective group homomorphism

$$
\psi_{\Gamma}: J(X) \rightarrow \operatorname{Hom}(\Gamma, \mathbf{U}),
$$

where $\mathbf{U}$ is the group of complex numbers of modulus 1 .

The groups $\Gamma_{0}(N, D)$ and $\Gamma_{1}(N, D)$ can be regarded as discrete subgroups of $\mathrm{SL}_{2}(\mathbf{R})$ (via $i_{\infty}$ ) of finite covolume. With $\Gamma=\Gamma_{0}(N, D)$, we obtain from (3) the injection

$$
\psi: J_{0}(N, D) \rightarrow \operatorname{Hom}\left(\Gamma_{0}(N, D), \mathbf{U}\right),
$$

and that an element $x \in J_{0}(N, D)$ belongs to the Shimura subgroup $\Sigma(N, D)$ if and only if the kernel of $\psi(x)$ contains $\Gamma_{1}(N, D)$. Using the identification (2), we deduce from $\psi$ a canonical injection

$$
\psi^{\prime}: \Sigma(N, D) \rightarrow \operatorname{Hom}\left((\mathbf{Z} / N \mathbf{Z})^{\times}, \mathbf{U}\right) .
$$

Furthermore, a homomorphism $h: \Gamma_{0}(N, D) \rightarrow \mathbf{U}$ belongs to the image of $\psi$ if and only if its kernel contains all the elliptic elements of $\Gamma_{0}(N, D)$.

From [4, IV 3A], the elliptic elements of $\Gamma_{0}(N, D)$ can only be of order 2 or 3 , i.e., they satisfy either $x^{2}+1=0$ or $x^{2}+x+1=0$. There are elliptic elements of order 2 (resp. 3 ) if and only if $(-4 / l) \neq 1$ (resp. $(-3 / l) \neq 1)$ for all $l \mid D$. Consequently, a homomorphism $g:(\mathbf{Z} / N \mathbf{Z})^{\times} \rightarrow \mathbf{U}$ belongs to the image of $\psi^{\prime}$ if and only if its kernel contains $S^{\prime}$, where $S^{\prime}$ contains -1 and the roots in $\mathbf{Z} / N \mathbf{Z}$ of the polynomials $X^{2}+t X+1=0$ for

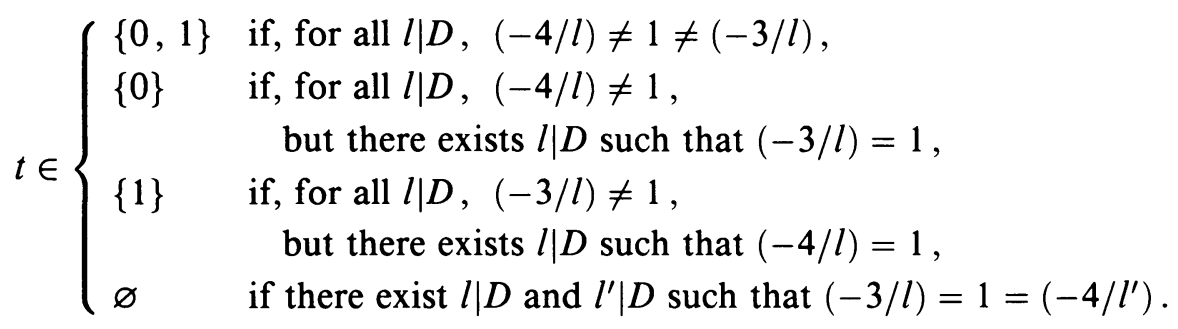

This proves Theorem 1 .

\section{ORDERS AND EXPONENTS OF $\Sigma(N, D)$}

2.1. Order of $\Sigma(N, D)$. Let $\mu_{2}(\mathbf{Z} / N \mathbf{Z})$ and $\mu_{3}(\mathbf{Z} / N \mathbf{Z})$ denote the groups of square roots and cube roots of unity in $\mathbf{Z} / N Z$, respectively. We make use of the two following lemmas to determine the order of $\Sigma(N, D)$.

Lemma 1. Assume that there exists $a \in \mathbf{Z} / N \mathbf{Z}$ such that $a^{2}+1=0$. The subgroup of $(\mathbf{Z} / N \mathbf{Z})^{\times}$generated by the roots of $X^{2}+1$ is equal to $\mu_{2}(\mathbf{Z} / N \mathbf{Z}) \cup$ $\mu_{2}(\mathbf{Z} / N \mathbf{Z}) a$; it contains -1 . The order of this group is $2^{k+1}$ (where $k$ is as in Corollary 1).

Lemma 2. Assume that $X^{2}+X+1$ has a root in $\mathbf{Z} / N \mathbf{Z}$. The subgroup of $(\mathbf{Z} / N \mathbf{Z})^{\times}$generated by these roots is then equal to $\mu_{3}(\mathbf{Z} / N \mathbf{Z})$. The order of this group is $3^{k}$.

The above are Lemmas 2 and 3 in [2]. The proofs are given there.

Corollary 1 now follows immediately from Theorem 1 and Lemmas 1 and 2 . 
2.2. Exponent of $\Sigma(N, D)$. Let $e$ be the exponent of $\Sigma(N, D)$ and let $N \geq$ 5 . We prove Corollary 2 by considering the two following cases:

(a) When $m_{2}=2$. In this case, $N$ is of the form $N^{\prime}$ or $2 N^{\prime}$, with $N^{\prime} \neq$ 1 and each prime factor of $N^{\prime}$ congruent to $1 \bmod 4 m_{3}$, and $\Sigma(N, D)$ is isomorphic to the quotient of the group

$$
A=\prod_{p \mid N^{\prime}}\left(\left(\mathbf{Z} / \frac{p-1}{2 m_{3}} \mathbf{Z}\right) \times\left(\mathbf{Z} / p^{r^{\prime}} \mathbf{Z}\right)\right)
$$

by the unique subgroup of order 2 that has a nonzero projection on each factor $\mathbf{Z} /\left((p-1) / 2 m_{3}\right) \mathbf{Z}$. If $N^{\prime}$ has at least two distinct prime factors $\left(m_{1}=1\right)$, then $e$ is equal to $e_{A}$, the exponent of $A$. If $N^{\prime}$ is a prime power $\left(m_{1}=2\right)$, then $e=e_{A} / 2$. Consequently,

$$
e=\frac{e_{A}}{m_{1}}=\frac{e_{0}}{m_{1} \cdot 2 m_{3}}=\frac{e_{0}}{m_{1} m_{2} m_{3}} .
$$

(b) When $m_{2}=1$. In this case, the subgroup $\Sigma(N, D)$ is isomorphic to $(\mathbf{Z} / N \mathbf{Z})^{\times} /\{ \pm 1\} \mu_{m_{3}}(\mathbf{Z} / N \mathbf{Z})$. If $m_{3}=1$, then $e=e_{0} / m_{1}$ since the exponent of $(\mathbf{Z} / N \mathbf{Z})^{\times} /\{ \pm 1\}$ is $e_{0} / m_{1}$. If $m_{3}=3$, then $2 \nmid N$ and an argument similar to the one in (a) shows that $e=e_{0} / 3 m_{1}=e_{0} / m_{1} m_{3}$. Therefore, we have $e=e_{0} / m_{1} m_{2} m_{3}$.

2.3. The case where $e=1$. For each odd prime divisor $p$ of $N$, the exponent $r_{p}$ of $p$ in the prime power decomposition of $N$ is at most 1 ; otherwise, $p$ would divide $e$. Moreover, $p-1$ divides $m_{1} m_{2} m_{3} e$ and a fortiori 12 , hence $p \in\{3,5,7,13\}$. Furthermore, the exponent $r_{2}$ of 2 is at most 2 ; otherwise 2 would divide $e$.

The integer $N$ cannot be divisible by $2 \cdot 7,2 \cdot 13,5 \cdot 7$, or $5 \cdot 13$ since $m_{3}=1$ and $3 \mid e$ in these cases. It is not divisible by $3 \cdot 5,3 \cdot 7,3 \cdot 13,7 \cdot 13$, $2^{2} \cdot 3$, or $2^{2} \cdot 5$ since $2 \mid e$ in these cases. Hence, $N$ can only be $1-7,10$, and 13.

For $1 \leq N \leq 4$ and $N=6$, we have $\Sigma(N, D)=\{1\}$ from Corollary 1 .

When $N=5$, we have $e_{0}=4, m_{1}=2$, and $m_{3}=1$. We need $m_{2}=2$ to ensure $e=1$. This means that $(-4 / l) \neq 1$ for all $l$ dividing $D$.

When $N=7$, we have $e_{0}=6, m_{1}=2$, and $m_{2}=1$. In order to have $e=1$, we need $m_{3}=3$, which is the case provided $(-3 / l) \neq 1$ for all $l$ dividing $D$.

When $N=10$, we have $e_{0}=4, m_{1}=2$, and $m_{3}=1$. To have $e=1$, we require $m_{2}=2$, which is true when $(-4 / l) \neq 1$ for all $l$ dividing $D$.

When $N=13$, we have $e_{0}=12$ and $m_{1}=2$. We need $m_{2}=2$ and $m_{3}=3$ so that $e=1$. This means that $(-4 / l) \neq 1$ and $(-3 / l) \neq 1$ for all $l$ dividing $D$.

This completes the proof of Corollary 3 .

2.4. Behaviour of exponent when $N$ approaches infinity. It is clear from Corollary 2 that if the exponent of $\Sigma(N, D)$ is bounded then the prime divisors of $N$ are bounded and so are the exponents of the prime divisors. Hence, the set of positive integers $N$ for which the exponent of $\Sigma(N, D)$ is bounded is finite. This means that $e$ tends to infinity as $N$ approaches infinity. 


\section{ACKNOWLEDGMENT}

The author wishes to thank Ken Ribet for helpful conversations while working on this project.

\section{REFERENCES}

1. B. Jordan, On the diophantine arithmetic of Shimura curves, Ph.D. thesis, Harvard University, 1981.

2. S. Ling and J. Oesterlé, The Shimura subgroup of $J_{0}(N)$, Astérisque 196-197 (1991), 171203.

3. B. Mazur, Modular curves and the Eisenstein ideal, Inst. Hautes. Études. Sci. Publ. Math. 47 (1978), 33-186.

4. M.-F. Vignéras, Arithmétique des algèbres de quaternions, Lecture Notes in Math., vol. 800, Springer-Verlag, Berlin and New York, 1980.

Department of Mathematics, University of California, Berkeley, California 94720

Current address: Department of Mathematics, National University of Singapore, Singapore 0511, Singapore

E-mail address: matlings@nuscc.nus.sg 